\title{
Evaluation of an Improved Technique for Geosynthetic-Reinforced and Pile-Supported Embankment
}

\author{
Jun Zhang, ${ }^{1,2}$ Shao-wen Liu, ${ }^{1}$ and He-fu $\mathrm{Pu}^{3}$ \\ ${ }^{1}$ Key Laboratory of Highway Construction and Maintenance Technology in Loess Region, \\ Shanxi Transportation Research Institute, Taiyuan 030006, China \\ ${ }^{2}$ Key Laboratory of Transportation Tunnel Engineering, Ministry of Education, Chengdu 610031, China \\ ${ }^{3}$ Department of Civil, Architectural and Environmental Engineering, Missouri University of Science and Technology, \\ Rolla, MO 65409, USA \\ Correspondence should be addressed to Jun Zhang; zhangjun_hust@qq.com
}

Received 1 May 2015; Revised 27 June 2015; Accepted 28 June 2015

Academic Editor: João M. P. Q. Delgado

Copyright (C) 2015 Jun Zhang et al. This is an open access article distributed under the Creative Commons Attribution License, which permits unrestricted use, distribution, and reproduction in any medium, provided the original work is properly cited.

With a large number of applications of conventional technique for geosynthetic-reinforced and pile-supported (GRPS) embankment (called CT embankment), many deficiencies have been exposed. In view of the deficiencies, an improved technique, fixed-geosynthetic-reinforced and pile-supported embankment (called FGT embankment), is developed. To investigate the performance of the FGT embankment, the comparison analyses and parametric studies are conducted by Finite Element Method (FEM). The influencing factors investigated include elastic modulus of soil, tensile stiffness of geosynthetics, pile length, pile spacing, and pile elastic modulus. In addition, the cost evaluation for the FGT embankment and CT embankment is also made. The results show that the FGT embankment can significantly reduce the settlement and differential settlement, enhance the stability, and provide an economical and effective measure for the construction of high embankment at the bridge approach.

\section{Introduction}

With a large number of applications of conventional technique for geosynthetic-reinforced and pile-supported (GRPS) embankment (called CT embankment), many deficiencies have been exposed [1-3]. For example, the efficacy of controlling the settlement and differential settlement is insufficient, and the efficiency of geosynthetics may not be fully mobilized. The differential settlement in particular is still great when using the CT embankment at the bridge approach. This differential settlement could be attributed to the stiffness difference between the bridge abutment and bridge approach embankment [4-6]. A small differential settlement can produce a significant vehicle bump at the end of the bridge and this vehicle bump can lead to higher vehicle or bridge maintenance costs and discomfort to drivers and passengers $[7,8]$.
In view of these deficiencies, an improved technique, fixed-geosynthetic-reinforced and pile-supported embankment (called FGT embankment), is developed and introduced herein. The structures for the CT and FGT embankments are presented in Figure 1. In the FGT embankment, the geosynthetic is fixed on the pile caps, and this fixed system is comprised of a steel bar fulcrum and concrete fixed top. In comparison with the CT embankment, the numerical analysis and cost evaluation are carried out to investigate the performance of FGT embankment in this study.

\section{Numerical Analysis}

2.1. Numerical Modeling. The following studies are on the basis of a bridge approach in Chang-An Expressway (Figure 2), which is located at the east of MCK +826 number 0 Bridge in Changzhi City, central region of China. Taking 
TABLE 1: Material parameters used in the numerical simulations.

\begin{tabular}{|c|c|c|c|c|c|c|c|}
\hline Material & $E /(\mathrm{MPa})$ & $\gamma /\left(\mathrm{kN} / \mathrm{m}^{3}\right)$ & $c /(\mathrm{kPa})$ & $\varphi /\left(^{\circ}\right)$ & $v$ & $R_{\text {inter }}$ & $H / L /(\mathrm{m})$ \\
\hline Embankment fill & 20.0 & 19.0 & 16.8 & 24.6 & 0.30 & 0.50 & 5.0 \\
\hline Silty clay & 5.2 & 17.2 & 14.2 & 18.3 & 0.33 & 0.65 & 3.5 \\
\hline Silty soil & 7.6 & 17.8 & 7.6 & 19.7 & 0.33 & 0.65 & 6.0 \\
\hline Gravelly sand & 30.0 & 20.1 & 0 & 28.6 & 0.30 & 0.65 & 4.0 \\
\hline Clay & 22.1 & 18.3 & 15.7 & 21.0 & 0.30 & 0.80 & 6.5 \\
\hline
\end{tabular}

Note: $E$ is elastic modulus; $\gamma$ is unit weight; $\nu$ is Poisson's ratio; $c$ is cohesive strength; $\varphi$ is friction angle; $H$ is height of embankment fill; and $L$ is thickness of soil layers.

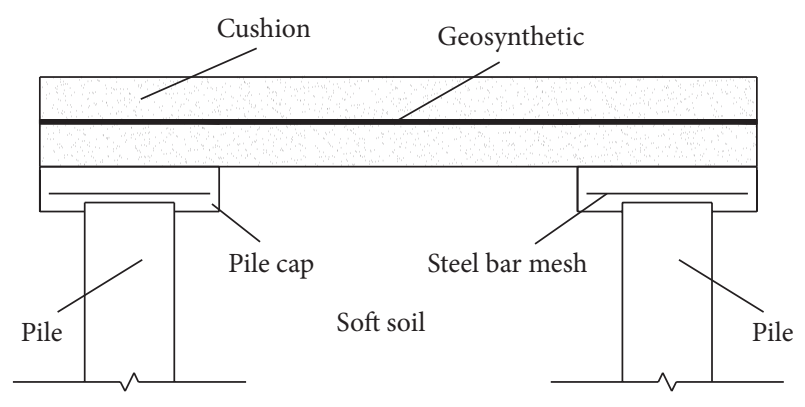

(a) CT structure

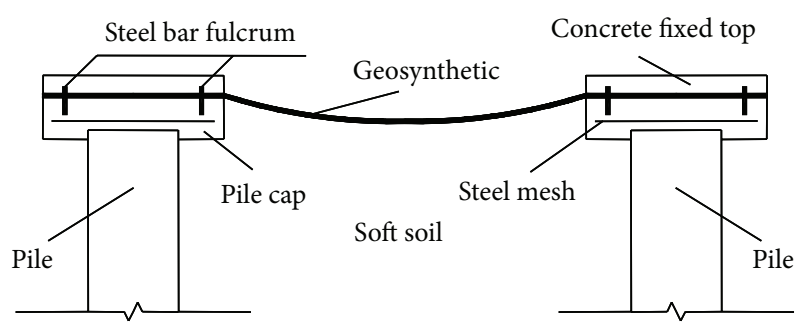

(b) FGT structure

FIGURE 1: Illustration of CT and FGT structures [3].

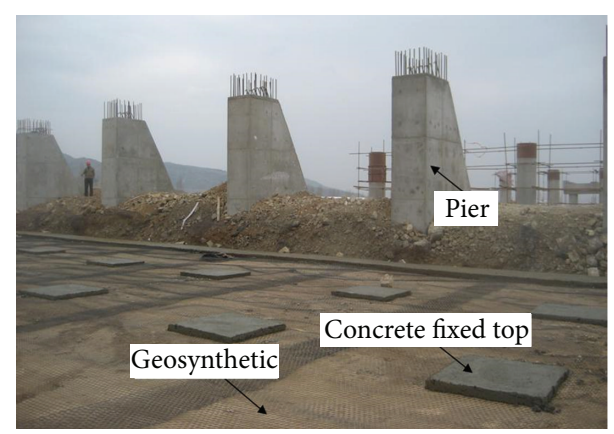

FIgURE 2: Bridge approach at MCK + 826 number 0 Bridge.

this project as a prototype, a two-dimensional finite element model is established using the software package PLAXIS. Due to symmetry of the embankment, half of the embankment cross section is modeled in PLAXIS. The parameters values used in the numerical simulations, shown in Table 1, are obtained from the in situ and laboratory tests. To convert the three-dimensional problem into a two-dimensional one, the equivalent elastic modulus of pile in the two-dimensional

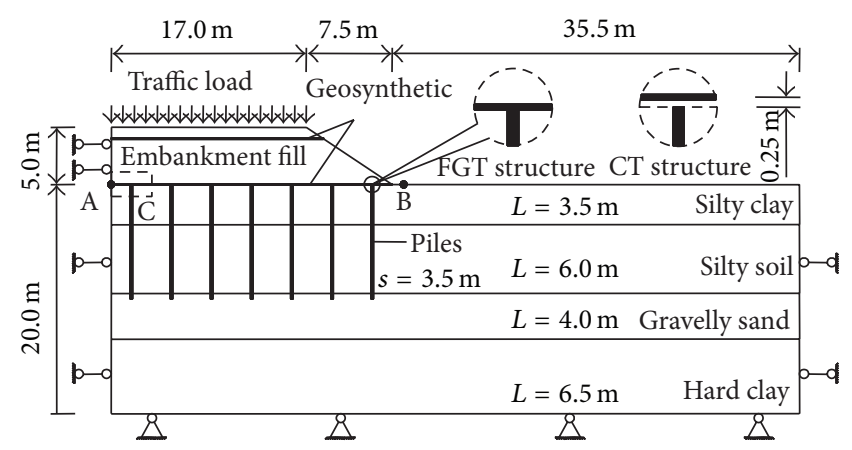

FIGURE 3: Dimensions and boundary conditions in numerical model.

model is calculated as $E_{\text {eq }}=E_{p} m+E_{s}(1-m)$, where $E_{p}$ and $E_{s}$ are the elastic modulus of the pile and the soil, respectively; and $m$ is the area replacement ratio [9]. The geometry and boundary conditions of the model are the same as those presented by Zhang et al. [3].

The dimensions and the boundary conditions in the numerical model are presented in Figure 3. The boundary condition at the bottom is assumed to be completely rigid and the vertical boundaries are fixed against horizontal displacement but allowed to move in the vertical direction. The subgrade and embankment fill are modeled as linearly elastic-plastic materials with Mohr-Coulomb failure criterion. The piles and geosynthetic are modeled as linearly elastic materials.

In the numerical modeling, the 15-node triangular elements (Figure 4(a)) are used to model soil layers and embankment fill. The 15-node triangle is the most accurate element in the PLAXIS and can provide a high accuracy results for complex problems. The 5-node beam (geogrid) elements (Figure 4(b)) and five pairs of interface elements (Figure 4(c)) are used together with 15-node triangular elements. The soil-structure interface is modeled as elasticplastic. In elastic region, the shear stress at the interface is given by

$$
|\tau|<\sigma_{n} \tan \varphi^{\prime}+c^{\prime} .
$$

In plastic region, the shear stress at the interface is given by

$$
|\tau|=\sigma_{n} \tan \varphi^{\prime}+c^{\prime},
$$

where $\sigma_{n}$ is normal stress and $\varphi^{\prime}$ and $c^{\prime}$ are friction angle and cohesive strength of the subgrade-structure interface, 


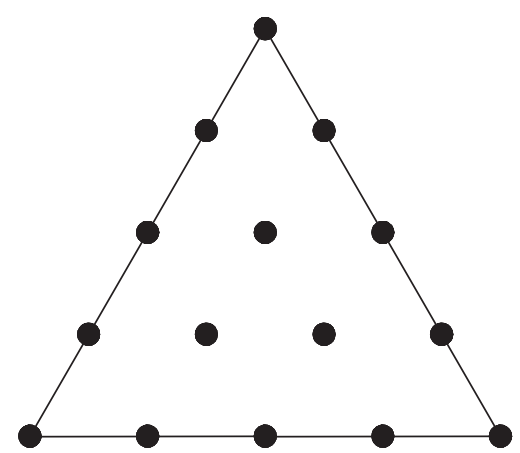

(a) 15-node triangular elements

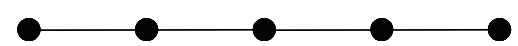

(b) 5-node beam (geogrid) elements

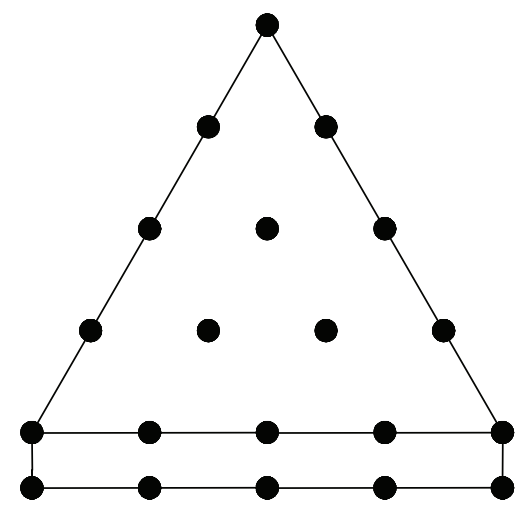

(c) Five pairs of interface elements

FIGURE 4: Basic elements used in the numerical model.

respectively. The strength properties of interfaces can be determined from the subgrade (embankment) properties with a correlated strength reduction factor, $R_{\text {inter }}$, and are calculated as

$$
\begin{aligned}
\tan \varphi^{\prime} & =R_{\text {inter }} \tan \varphi_{\text {soil }}, \\
c^{\prime} & =R_{\text {inter }} c_{\text {soil }},
\end{aligned}
$$

where $\varphi_{\text {soil }}$ and $c_{\text {soil }}$ are friction angle and cohesive strength of the subgrade soil [10].

2.2. Model Calibration. A model calibration was conducted to ensure that the numerical modeling is reasonable and representative of the field conditions [3]. The settlements at the centerline of the ground surface (Point $\mathrm{A}$ as shown in Figure 3) and lateral displacements at $1.0 \mathrm{~m}$ away from the embankment toe (Point B as shown in Figure 3) in the CT and FGT cases are measured by the settlement gauges and total station instrument, respectively. Figure 5 shows that the simulation results exhibit a reasonable agreement with the field measurement data. The pile efficacy, which is the ratio of the embankment load on a single pile to the total embankment load over the tributary area of the pile below the geosynthetic, changed with the embankment height as shown in Figure 6. The calculated results are again shown to be in good agreement with field measured data. The above model calibration forms the basis for the following study.

2.3. Results and Comparisons. The settlement profiles at embankment crest for both cases are presented in Figure 7. The settlement profile for the FGT embankment is similar to that for the CT embankment. However, the settlement for the FGT embankment is much smaller than that for the CT embankment. The maximum settlement for the FGT embankment and CT embankment is $75.1 \mathrm{~mm}$ and $83.5 \mathrm{~mm}$, respectively.

Stability is one of the most important issues for high embankment [11-13]. As an important control parameter for the stability of high embankment [14], the lateral displacement is discussed herein. Figure 8 shows that the lateral displacement at the embankment toe varies with depth. As expected, the lateral displacement for the CT embankment is greater than that for the FGT embankment. The maximum lateral displacements for the FGT embankment and CT embankment are 18.6 and $22.9 \mathrm{~mm}$, respectively. The maximum lateral displacement for the FGT embankment is reduced by $18.8 \%$ as compared to that for the CT embankment. The FGT embankment can significantly reduce the lateral displacement and thus improve the embankment stability.

Figure 9 shows that the vertical displacements of geosynthetic display different responses for both cases. The vertical displacement for the CT embankment is shown to be larger than that for the FGT embankment. However, the differential vertical displacements between the valley and adjacent heave for the FGT embankment are much larger than those for the CT embankment. Below the embankment crest, the maximum differential vertical displacement for the FGT embankment is $32.1 \mathrm{~mm}$ and is more than 3 times larger than that for the CT embankment.

The maximum tension of geosynthetic is an important index for evaluating the efficiency of geosynthetic [15]. As shown in Figure 10, the maximum tension in both cases increases with increasing embankment height. For all the embankment heights, the maximum tension for the FGT embankment is greater than that for the CT embankment. In addition, the difference of the maximum tension between the two cases tends to increase with increasing embankment height. Thus, the FGT embankment can better mobilize the geosynthetic efficiency for all the embankment height considered.

The distributions of lateral displacement at the top of the piles for both cases are presented in Figure 11. The lateral displacements for both cases generally increase with the distance from the embankment centerline. The lateral displacement curve for the FGT embankment is smoother than that for the CT embankment. It can be concluded that the FGT embankment can reduce the lateral displacement at the top of the piles and improve the interaction between the piles.

The variation of maximum bending moment of piles with distance from embankment centerline is presented in Figure 12. In general, the maximum bending moments for the FGT embankment are greater than those for the CT 


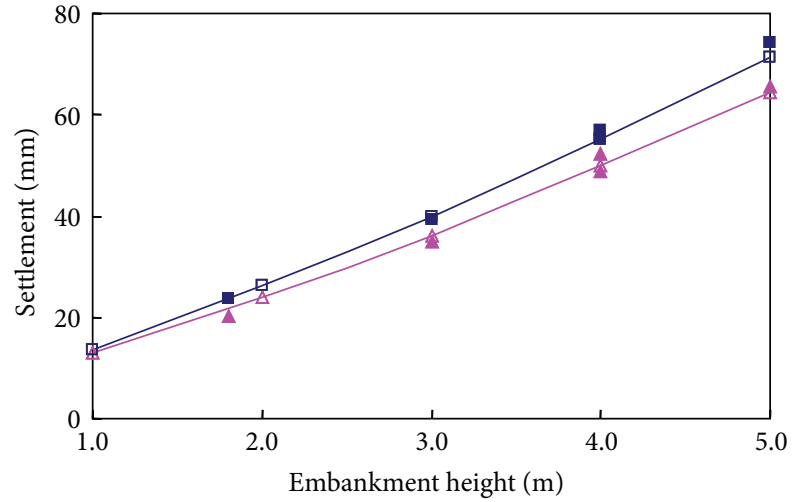

$\begin{array}{ll}\triangle & \text { FGT (calculated) } \\ \text { FGT (measured) } & \square\end{array}$

(a) Settlement at centerline of ground surface

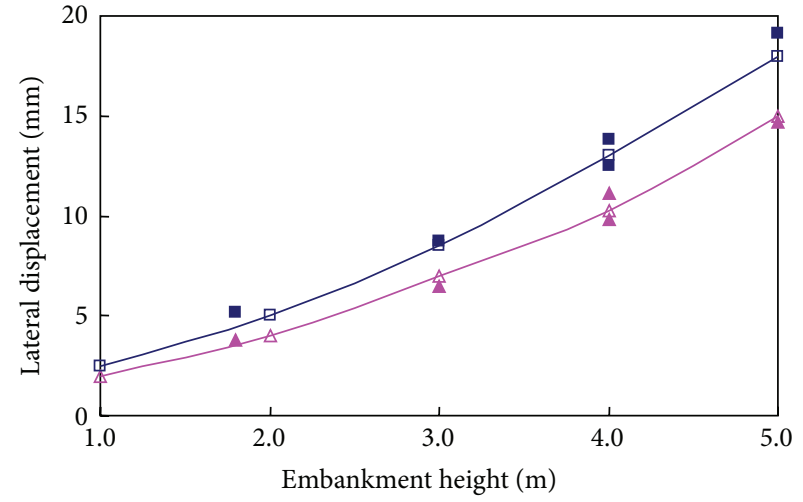

$\begin{array}{ll}\triangle & \text { FGT (calculated) } \\ \text { FGT (measured) } & \square \text { CT (calculated) }\end{array}$

(b) Lateral displacement at $1.0 \mathrm{~m}$ away from embankment toe

Figure 5: Comparisons of numerical results with field measurements.

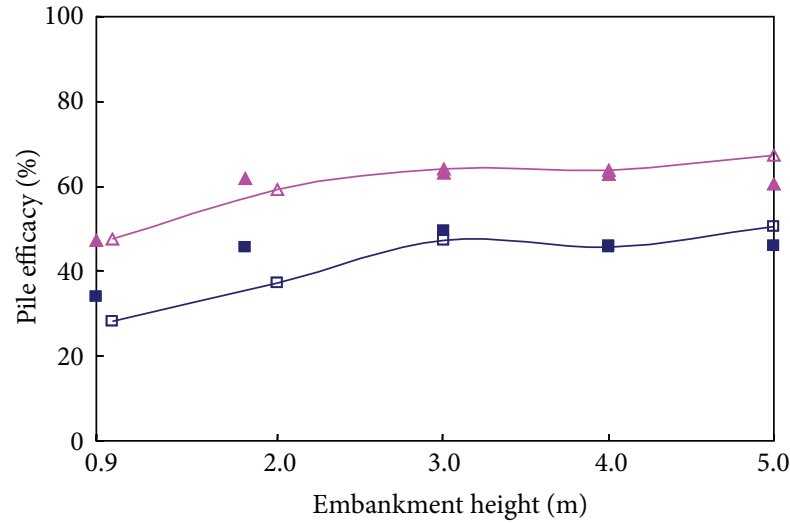

$\triangle$ FGT (calculated)

- FGT (measured)

๑- CT (calculated)

- CT (measured)

FIGURE 6: Variation of pile efficacy below geosynthetic with embankment height.

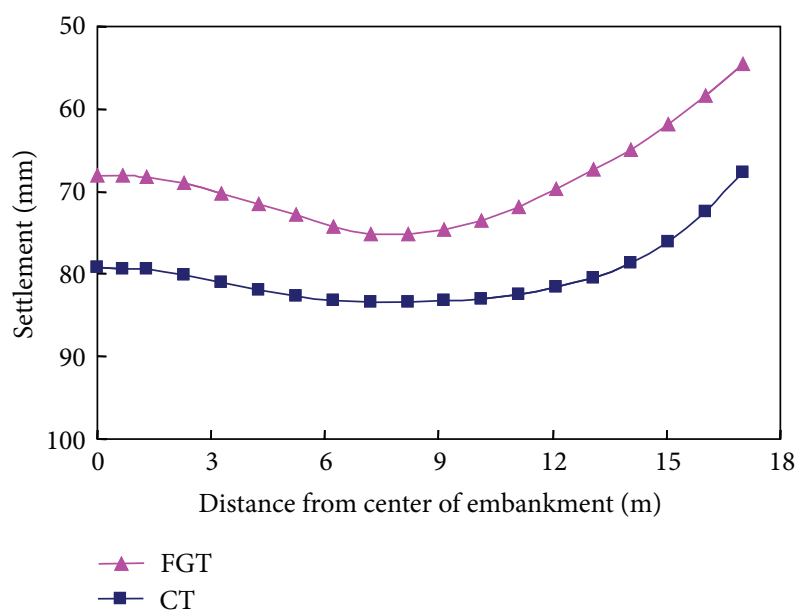

Figure 7: Settlement at embankment crest.

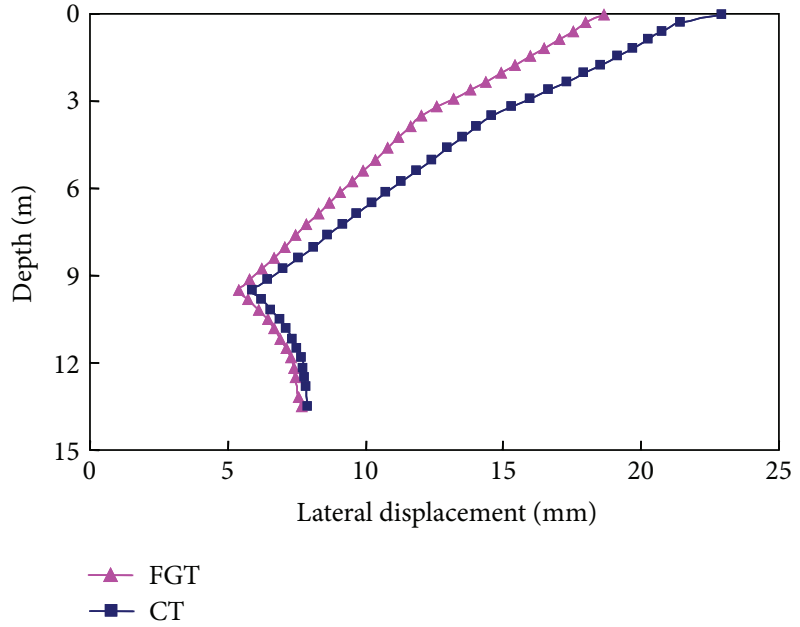

FIGURE 8: Variation of lateral displacement with depth.

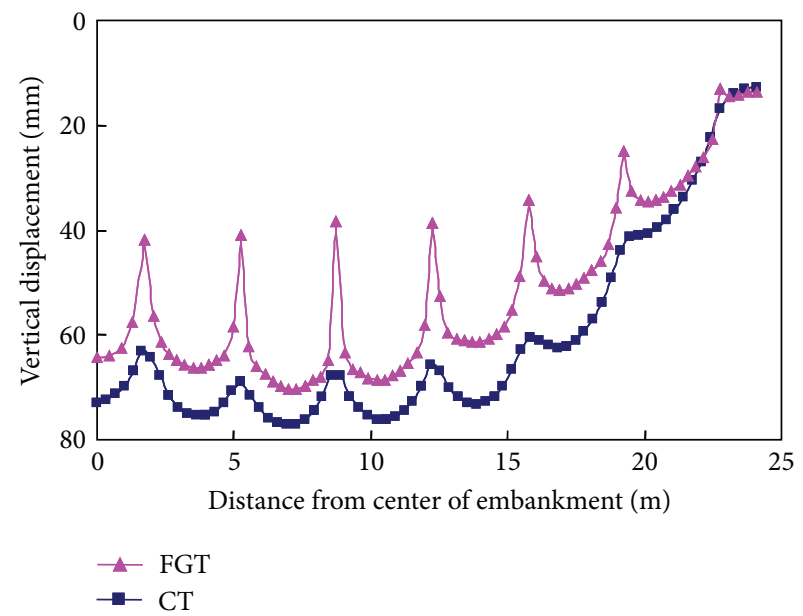

Figure 9: Vertical displacement of geosynthetic. 


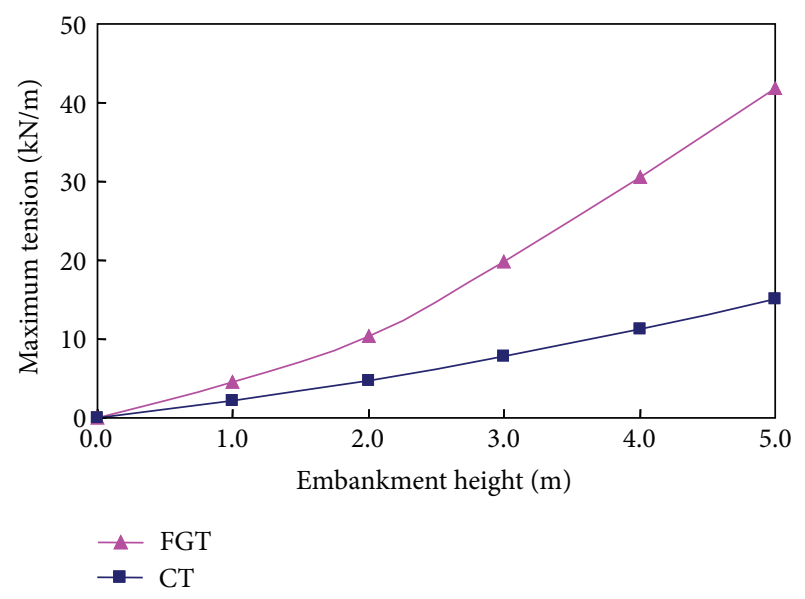

FIgURE 10: Variation of maximum tension of geosynthetic with embankment height.

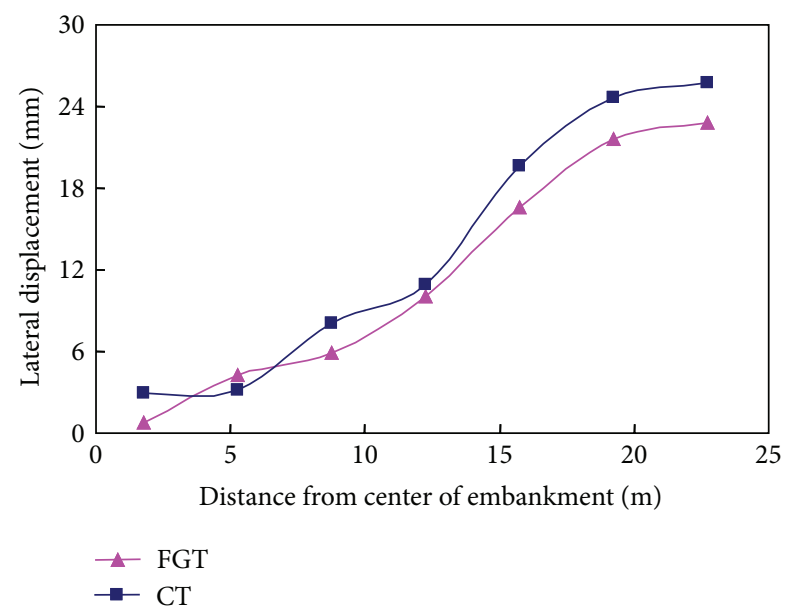

FIGURE 11: Variation of lateral displacement at pile top with distance from embankment centerline.

embankment. In addition, the distribution of maximum bending moment for the FGT embankment is more uniform relative to the CT embankment. The maximum bending moment among the piles for the CT embankment is 1.3 times that for the FGT embankment. The FGT embankment is shown to improve the interaction between the piles.

2.4. Influencing Factors. To further investigate the performance of the FGT embankment, five key influencing factors are selected for parametric study. The influencing factors include elastic modulus of soil, tensile stiffness of geosynthetic, pile length, pile spacing, and pile elastic modulus. In the parametric study, the FGT embankment and CT embankment mentioned above are the two baseline cases. The maximum settlement at the embankment crest, the lateral displacement at the embankment toe, and the maximum tension of geosynthetic are investigated.

The influence of elastic modulus of soil is studied by analyzing four cases with $0.6,0.8,1.0$, and 1.2 times of the elastic modulus of soil (including all soil layers) for

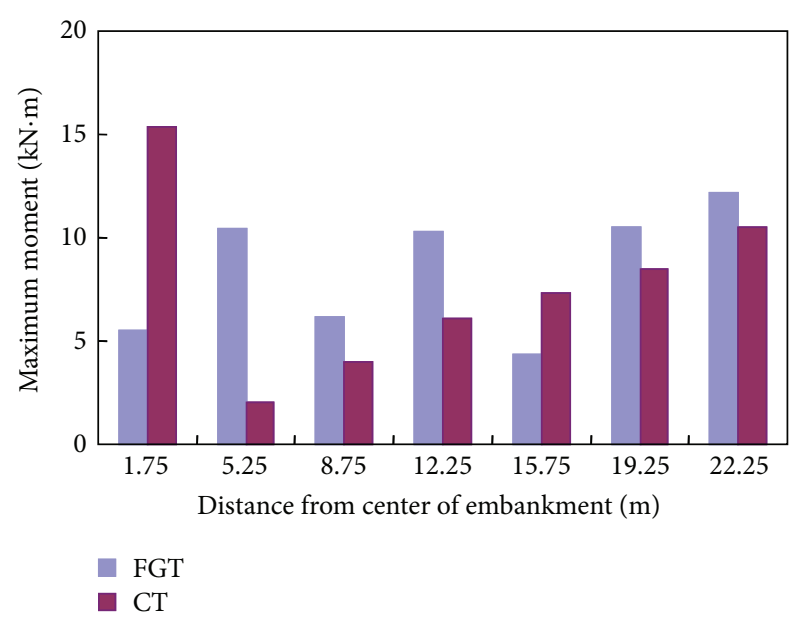

FIGURE 12: Variation of maximum bending moment of piles with distance from embankment centerline.

the baseline cases. As shown in Figure 13(a), the maximum settlement and lateral displacement decrease with increasing elastic modulus of soil. At a low elastic modulus (0.6 times of the elastic modulus of soil), the maximum settlement and lateral displacement for the FGT embankment are much smaller than those for the CT embankment. For the FGT embankment, the maximum settlement and lateral displacement are reduced by $19.1 \%$ and $10.1 \%$ as compared to the CT embankment, respectively. The differences of the maximum settlement and lateral displacement between the two cases decrease gradually with increasing elastic modulus of soil. The effect of elastic modulus of soil on the maximum tension of geosynthetic is presented in Figure 13(b). The maximum tension decreases with increasing elastic modulus of soil for the FGT embankment. However, the elastic modulus of soil has only slight influence on the maximum tension for the CT embankment. Thus, the FGT embankment is more competent for a soft soil condition.

Geosynthetic used for the GRPS embankment is expected to improve the pile efficiency and embankment stability by transferring load from the subgrade to the piles and reducing lateral displacement [16]. The influence of tensile stiffness of geosynthetic is studied by analyzing four cases with tensile stiffness of geosynthetic $=100,1000,10000$, and $100000 \mathrm{kN} / \mathrm{m}$. Figure 14(a) shows that the tensile stiffness of geosynthetic has a limited influence on the maximum settlement for both cases. However, the lateral displacement is reduced significantly with increasing tensile stiffness of geosynthetic. At the tensile stiffness of geosynthetic of $100 \mathrm{kN} / \mathrm{m}$, the lateral displacement for the FGT embankment is reduced by $16.1 \%$ as compared to the lateral displacement for CT embankment, and the corresponding reduction increases to $34.2 \%$ at the tensile stiffness of geosynthetic of $100000 \mathrm{kN} / \mathrm{m}$. As shown in Figure 14(b), the maximum tension increases with increasing tensile stiffness of geosynthetic. The maximum tension for the FGT embankment is greater than that for the CT embankment. In addition, the difference of the maximum tension between the two cases tends to be greater with increasing tensile stiffness of geosynthetic. 


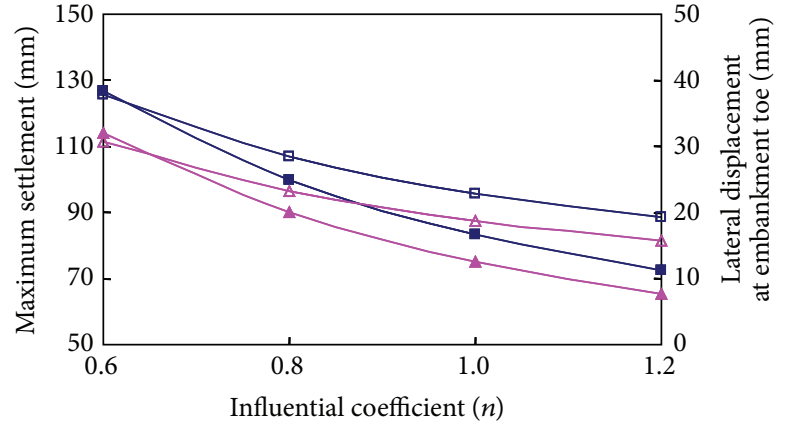

$\begin{array}{lll}\rightarrow \text { Maximum settlement-FGT } & \Delta \text { Lateral displacement-FGT } \\ \rightarrow-\text { Maximum settlement-CT } & \square \text { Lateral displacement-CT }\end{array}$

(a) Maximum settlement and lateral displacement

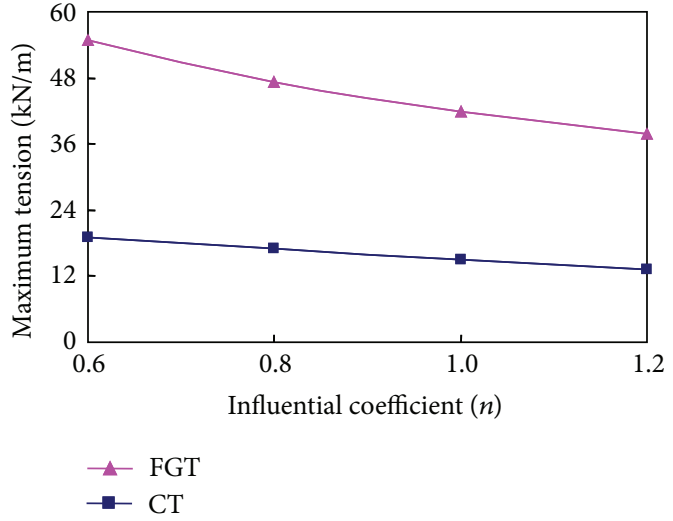

(b) Maximum tension

FIGURE 13: Influence coefficient of elastic modulus of soil.

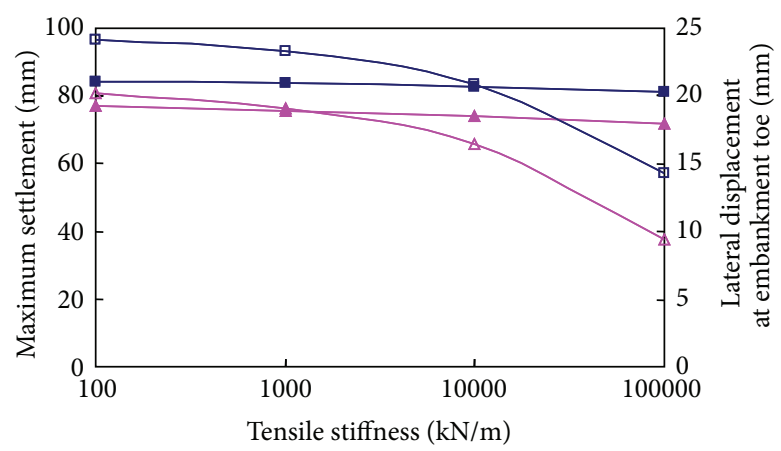

$\begin{array}{lll}\longrightarrow \text { Maximum settlement-FGT } & \triangle & \text { Lateral displacement-FGT } \\ \rightarrow \text { - Maximum settlement-CT } & \rightarrow & \text { Lateral displacement-CT }\end{array}$

(a) Maximum settlement and lateral displacement

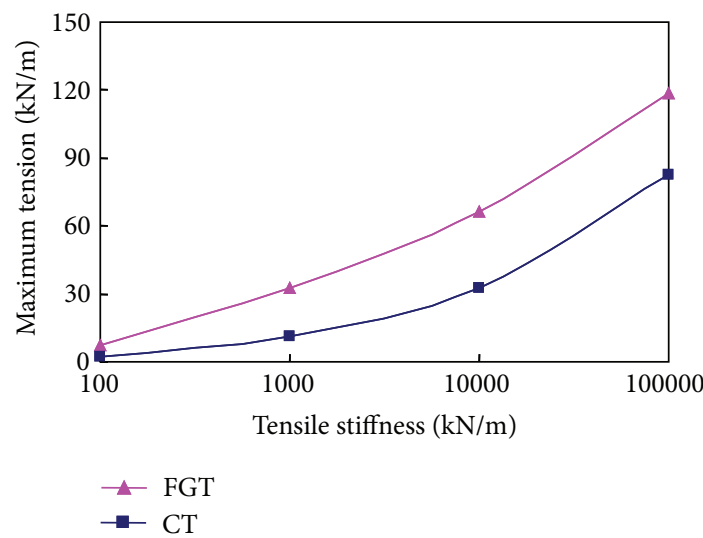

(b) Maximum tension

FIGURE 14: Influence of geosynthetic tensile stiffness.

The influence of pile length is studied by analyzing four cases with pile length of 6.0, 8.0, 10.0, and $12.0 \mathrm{~m}$. As shown in Figure 15(a), the maximum settlement and lateral displacement change with the pile length for both cases. As the pile length increases from 6.0 to $12.0 \mathrm{~m}$, the maximum settlement decreases only slightly at first but has a jump when the pile length increases from 8.0 to $10.0 \mathrm{~m}$. Then, the influence of pile length becomes less important again when the pile length exceeds $10.0 \mathrm{~m}$. Similarly, the degree of decrease on the lateral displacement becomes much sharper with the pile length increase from 8.0 to $10.0 \mathrm{~m}$. This phenomenon occurs because the piles are penetrated into firm soil layer. Figure 15(b) shows that the pile length has a significant influence on the maximum tension for the FGT embankment but limited influence for the CT embankment.

Pile spacing is an important design parameter for GRPS embankment. Closely spaced piles can transfer the surcharge load easily but are much uneconomical. A wide pile spacing, however, is likely to cause bearing capacity failure or slope stability failure [17]. In this study, the influence of pile spacing is studied by analyzing four cases with pile spacing of $2.5,3.0,3.5$, and $4.0 \mathrm{~m}$. The maximum settlement and lateral displacement increase with increasing pile spacing as presented in Figure 16(a). For both the FGT embankment and CT embankment, the maximum settlement and lateral displacement increase with increasing pile spacing. As shown in Figure 16(b), the maximum tension of geosynthetic for the FGT embankment firstly decreases and then increases with increasing pile spacing. In contrast, the maximum tension for the CT embankment firstly increases and then decreases. Overall, the effect of pile spacing on the maximum tension for the CT embankment is less important than that for the FGT embankment.

The influence of pile elastic modulus is studied by analyzing four cases with pile elastic modulus of $0.5,1.0,5.0$, and 10.0 MPa. As shown in Figure 17(a), the pile elastic modulus has a limited influence on the maximum settlement and lateral displacement for both cases. Similarly, Figure 17(b) shows that the maximum tensions of geosynthetic remain nearly constant with the increase of pile elastic modulus.

2.5. Discussion. The influence of a factor on the maximum settlement at the embankment crest, the lateral displacement 


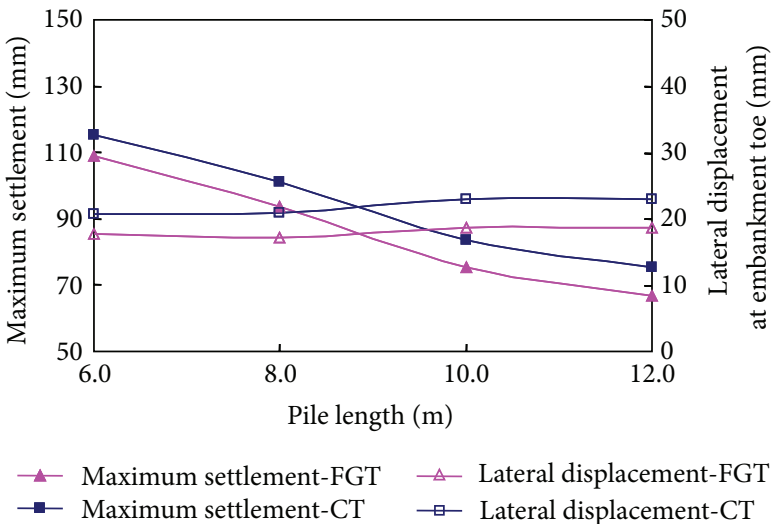

(a) Maximum settlement and lateral displacement

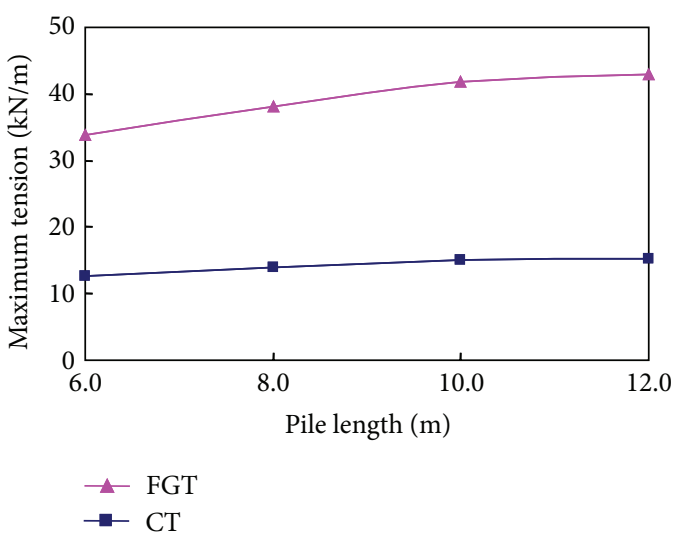

(b) Maximum tension

FIGURE 15: Influence of pile length.

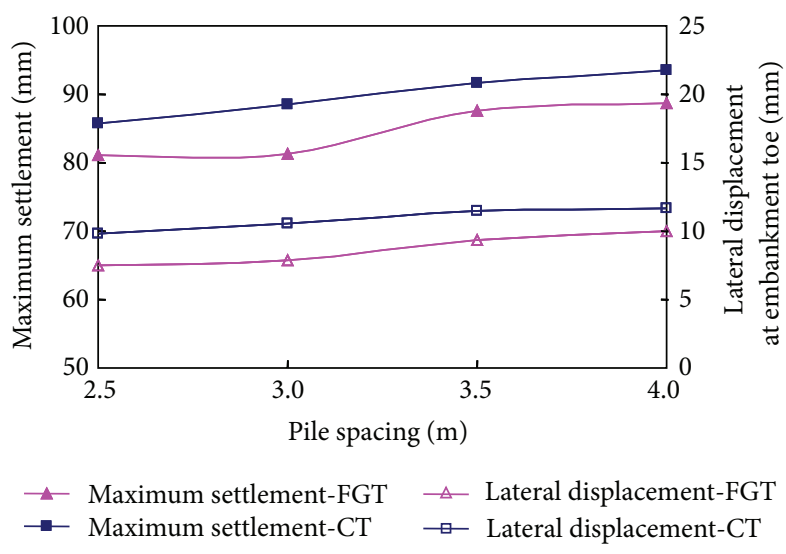

(a) Maximum settlement and lateral displacement

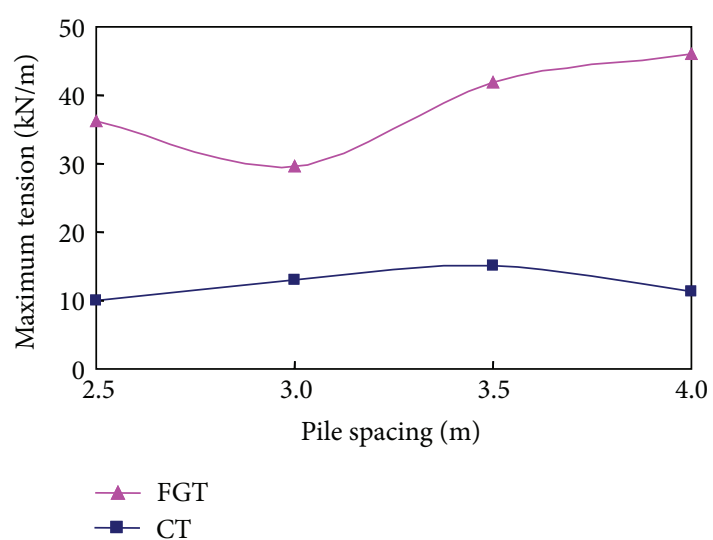

(b) Maximum tension

FIGURE 16: Influence of pile spacing.

at the embankment toe, and the maximum tension of geosynthetic is considered positive or negative based on its effect on the performance of FGT embankment. The positive and negative effects are defined as the decrease and increase of the performance of FGT embankment with an increase of the value of the factor, respectively. The degree of influence of the factor is defined as the change of the values relative to the mean values [18]. For example, the maximum settlements for the pile length of $6.0,8.0,10.0$, and $12.0 \mathrm{~m}$ are 109,94 , 75 , and $67 \mathrm{~mm}$, respectively. Thus, the degree of influence of the pile length on the maximum settlement is calculated as $(109-67) /[(109+67) / 2]=48 \%$. The degree of influence is divided into three grades: high (greater than 60\%), medium (between 30\% and 60\%), and low (less than 30\%). The detailed calculation method and the grade of the influence degree can be found in Huang and Han [18]. The degrees of influence for each factor are listed in Table 2 . The grades of the influence degree for each factor are presented in Table 3.

In this study, Tables 2 and 3 show that the pile length, pile spacing, and pile elastic modulus have a limited influence on the performance of FGT embankment. The elastic modulus of soil and tensile stiffness of geosynthetic have significant
TABLE 2: The degree of influence (\%).

\begin{tabular}{lccccc}
\hline Factors & $E$ & $E_{g}$ & $L_{p}$ & $D$ & $E_{p}$ \\
\hline$S_{\max }$ & 55 & 7 & 48 & 10 & 5 \\
$L_{\text {toe }}$ & 65 & 73 & 5 & 8 & 1 \\
$T_{\max }$ & 37 & 177 & 24 & 46 & 3 \\
\hline
\end{tabular}

Note: $S_{\max }$ is maximum settlement at embankment crest; $L_{\text {toe }}$ is lateral displacement at embankment toe; $T_{\max }$ is maximum tension; $E_{g}$ is tensile stiffness of geosynthetic; $L_{p}$ is pile length; $E_{p}$ is elastic modulus of pile; $D$ is pile spacing.

TABle 3: The grades of influence degree.

\begin{tabular}{lccccc}
\hline Factors & $E$ & $E_{g}$ & $L_{p}$ & $D$ & $E_{p}$ \\
\hline$S_{\max }$ & Medium (+) & Low (+) & Medium (+) & Low (-) & Low (+) \\
$L_{\text {toe }}$ & High (+) & High (+) & Low (-) & Low (-) & Low (-) \\
$T_{\max }$ & Medium (+) & High (-) & Low (-) & Medium (-) & Low (-) \\
\hline
\end{tabular}

influence and are the two important factors for the performance of FGT embankment. 


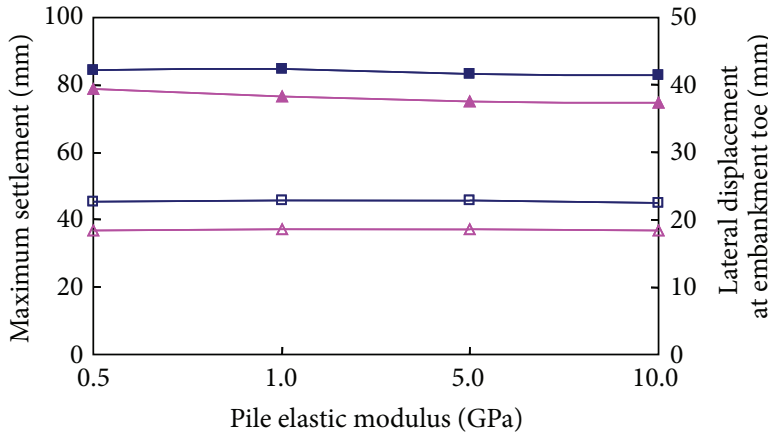

$\triangle$ Maximum settlement-FGT $\triangle$ Lateral displacement-FGT

$\rightarrow$ Maximum settlement-CT $\rightarrow$ Lateral displacement-CT

(a) Maximum settlement and lateral displacement

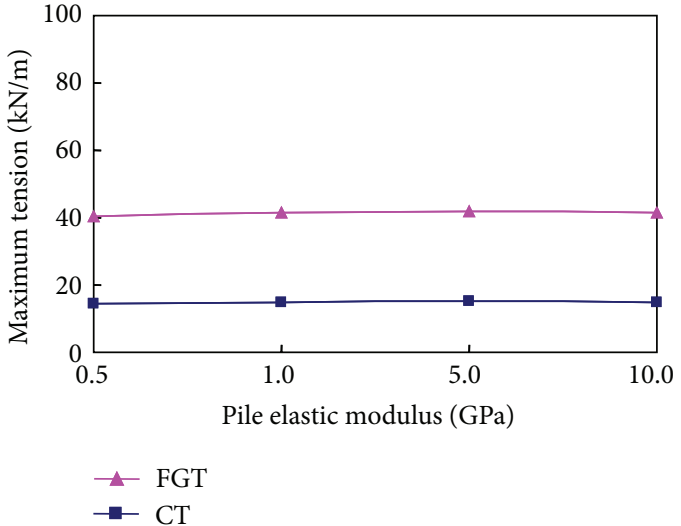

(b) Maximum tension

FIgURE 17: Influence of pile elastic modulus.

TABLE 4: Cost evaluation for CT and FGT embankments (RMB 1000 Yuan).

\begin{tabular}{|c|c|c|c|c|}
\hline & Labor cost & Material cost & Machinery cost & Total cost \\
\hline $\mathrm{CT}$ & 2120.6 & 2127.2 & 2011.7 & 6259.5 \\
\hline FGT & 2138.8 & 2271.0 & 2012.8 & 6422.6 \\
\hline Incremental cost & 18.2 & 143.3 & 1.1 & 163.1 \\
\hline Incremental rate & $0.9 \%$ & $6.8 \%$ & $0.1 \%$ & $2.6 \%$ \\
\hline
\end{tabular}

\section{Cost Evaluation}

In this study, the costs of ground improvement for the FGT embankment and CT embankment in the trial bridge approach embankment are compared. The costs include those for labor, materials, and machinery. The cost evaluation for the baseline case with embankment length of $1.0 \mathrm{~km}$ for the CT and FGT embankments is calculated and presented in Table 4.

For the FGT embankment, the total cost is increased by only $2.6 \%$ relative to the CT embankment. However, the maximum settlement at the embankment crest and the lateral displacement at the embankment toe for the FGT embankment are decreased by $10.1 \%$ and $18.8 \%$, respectively, relative to the CT embankment, as shown in Figures 7 and 8. Take the pile length and pile spacing; for example, the change rates of the cost and efficiency are presented in Table 5. The influence on the maximum settlement, lateral displacement, and maximum tension is considered positive or negative based on its effect on the performance of embankment as mentioned above. The FGT embankment is shown to provide an economical and effective measure for the construction of high embankment at the bridge approach.

\section{Conclusions}

In this paper, to improve the performance of the CT embankment at the bridge approach, the FGT embankment is pro-
TABLE 5: Change rates of the cost and efficiency.

\begin{tabular}{lccc}
\hline & FGT & Pile length-CT & Pile spacing-CT \\
\hline Baseline case $/(\mathrm{m})$ & - & 10.0 & 3.5 \\
Change case $/(\mathrm{m})$ & - & 12.0 & 3.0 \\
Total cost & $2.6 \%$ & $18.9 \%$ & $14.0 \%$ \\
Maximum settlement & $10.1 \%$ & $9.7 \%$ & $7.6 \%$ \\
Lateral displacement & $18.8 \%$ & $-0.5 \%$ & $0.8 \%$ \\
Maximum tension & $-178.4 \%$ & $-0.8 \%$ & $-13.8 \%$ \\
\hline
\end{tabular}

posed and studied. The numerical analysis and cost evaluation are conducted to investigate the performance of FGT embankment. From this study, the following conclusions can be drawn:

(1) The FGT embankment can significantly reduce the settlement and lateral displacement and improve load transfer from the subgrade to piles.

(2) The FGT embankment can significantly improve the embankment stability and the geosynthetic efficiency.

(3) In the FGT embankment, the average values of the maximum bending moment in the piles are greater than those in the CT embankment. However, the maximum bending moment among the piles is smaller than that in the CT embankment in this study.

(4) The pile length, pile spacing, and pile elastic modulus are shown to have limited influence on the performance of FGT embankment. The elastic modulus of soil and tensile stiffness of geosynthetic have significant influence on the performance of FGT embankment.

(5) The performance of the FGT embankment is improved significantly with only a slight increase of the total cost. Thus, the FGT embankment can provide an economical and effective measure for the construction of high embankment at the bridge approach. 


\section{Conflict of Interests}

The authors declare that there is no conflict of interests regarding the publication of this paper.

\section{Acknowledgments}

This work was supported by the National Natural Science Foundation of China (NSFC) (Grant no. 51278216), the Basic Research Program in Shanxi Province (Grant no. 2014021033-1), and the Research Fund of Key Laboratory of Transportation Tunnel Engineering, Ministry of Education (Grant no. TTE2014-05). The authors would like to express their gratitude for the financial support.

\section{References}

[1] G. S. Liu, L. W. Kong, and X. W. Li, "Analysis of treatment scheme for soft foundation on in expressway widening project and its verification," Chinese Journal of Rock Mechanics and Engineering, vol. 27, no. 2, pp. 309-315, 2008 (Chinese).

[2] K. Fei and H.-L. Liu, "Field test study and numerical analysis of a geogrid-reinforced and pile-supported embankment," Rock and Soil Mechanics, vol. 30, no. 4, pp. 1004-1011, 2009 (Chinese).

[3] J. Zhang, J. J. Zheng, and Y. E. Lu, "Evaluation of the new technique of geogrid-reinforced and pile-supported embankment at bridge approach," Journal of Bridge Engineering, vol. 19, no. 4, Article ID 06014001, 2014.

[4] R. M. Bakeer, M. A. Shutt, J. Q. Zhong, S. C. Das, and M. Morvant, "Performance of pile-supported bridge approach slabs," Journal of Bridge Engineering, vol. 10, no. 2, pp. 228-237, 2005.

[5] D. Q. Li and D. Davis, "Transition of railroad bridge approaches," Journal of Geotechnical and Geoenvironmental Engineering, vol. 131, no. 11, pp. 1392-1398, 2005.

[6] S. Roy and G. Thiagarajan, "Nonlinear finite-element analysis of reinforced concrete bridge approach slab," Journal of Bridge Engineering, vol. 12, no. 6, pp. 801-806, 2007.

[7] D. J. White, M. M. Mekkawy, S. Sritharan, and M. T. Suleiman, "'Underlying' causes for settlement of bridge approach pavement systems," Journal of Performance of Constructed Facilities, vol. 21, no. 4, pp. 273-282, 2007.

[8] H.-L. Zhang and C.-S. Hu, "Determination of allowable differential settlement in bridge approach due to vehicle vibrations," Journal of Bridge Engineering, vol. 12, no. 2, pp. 154-163, 2007.

[9] J. Huang, J. Han, and S. Oztoprak, "Coupled mechanical and hydraulic modeling of geosynthetic-reinforced columnsupported embankments," Journal of Geotechnical and Geoenvironmental Engineering, vol. 135, no. 8, pp. 1011-1021, 2009.

[10] PLAXIS 2D V8, Reference manual, 2002.

[11] M. De Souza Soares De Almeida, M. Ehrlich, A. P. Spotti, and M. E. S. Maroues, "Embankment supported on piles with biaxial geogrids," Proceedings of the Institution of Civil Engineers: Geotechnical Engineering, vol. 160, no. 4, pp. 185-192, 2007.

[12] S. W. Abusharar and J. Han, "Two-dimensional deep-seated slope stability analysis of embankments over stone columnsimproved soft clay," Engineering Geology, vol. 120, no. 1-4, pp. 103-110, 2011.

[13] T. Eskişar, J. Otani, and J. Hironaka, "Visualization of soil arching on reinforced embankment with rigid pile foundation using X-ray CT," Geotextiles and Geomembranes, vol. 32, pp. 44$54,2012$.

[14] S. Murugesan and K. Rajagopal, "Shear load tests on stone columns with and without geosynthetic encasement," Geotechnical Testing Journal, vol. 32, no. 1, pp. 76-85, 2009.

[15] J. Han and M. A. Gabr, "Numerical analysis of geosyntheticreinforced and pile-supported earth platforms over soft soil," Journal of Geotechnical and Geoenvironmental Engineering, vol. 128, no. 1, pp. 44-53, 2002.

[16] Y. M. Chen, W. P. Cao, and R. P. Chen, "An experimental investigation of soil arching within basal reinforced and unreinforced piled embankments," Geotextiles and Geomembranes, vol. 26, no. 2, pp. 164-174, 2008.

[17] J. Huang, Coupled mechanical and hydraulic modeling of geosynthetic-reinforced column-supported embankments [Ph.D. thesis], University of Kansas, Lawrence, Kan, USA, 2007.

[18] J. Huang and J. Han, "Two-dimensional parametric study of geosynthetic-reinforced column-supported embankments by coupled hydraulic and mechanical modeling," Computers and Geotechnics, vol. 37, no. 5, pp. 638-648, 2010. 

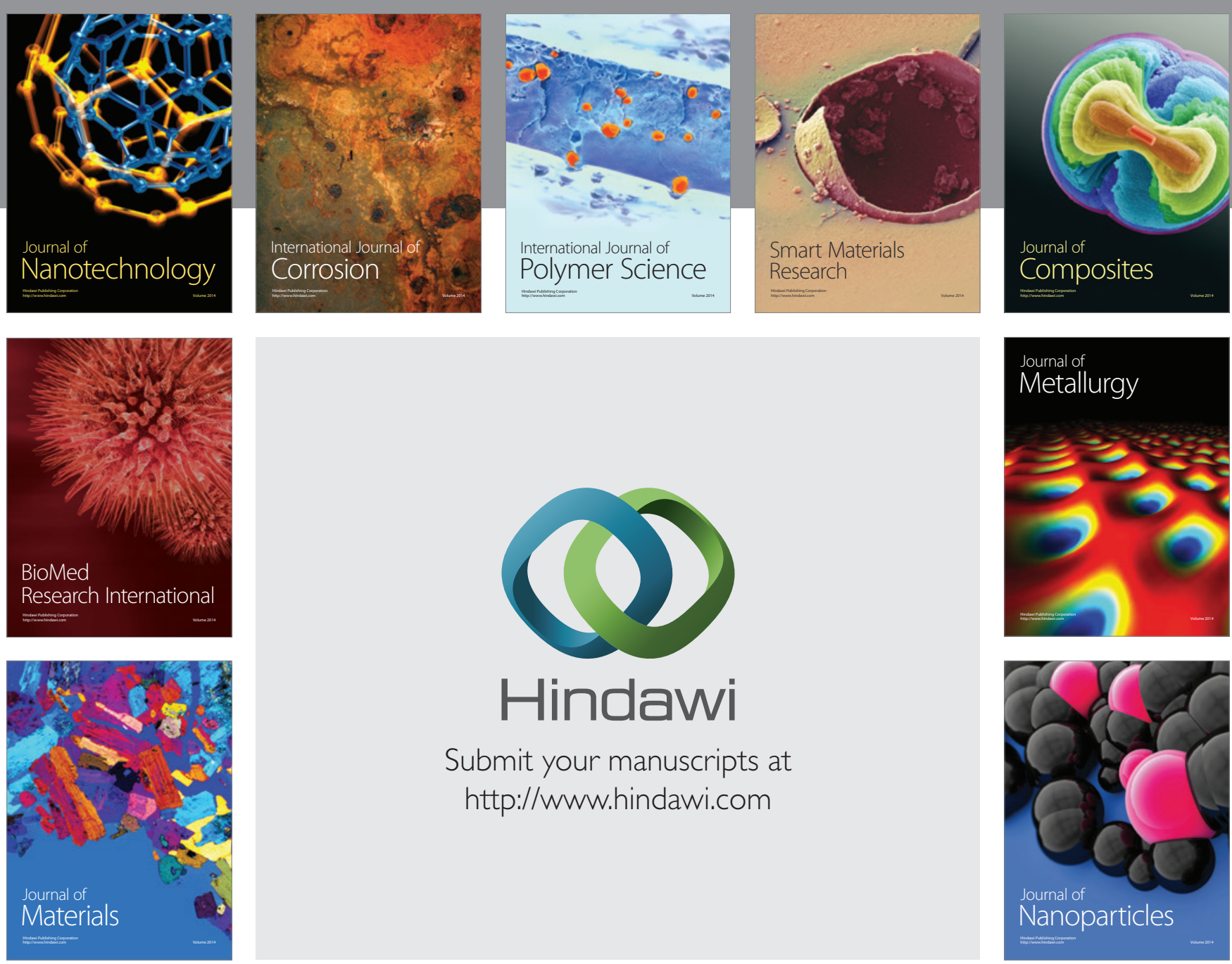

Submit your manuscripts at http://www.hindawi.com
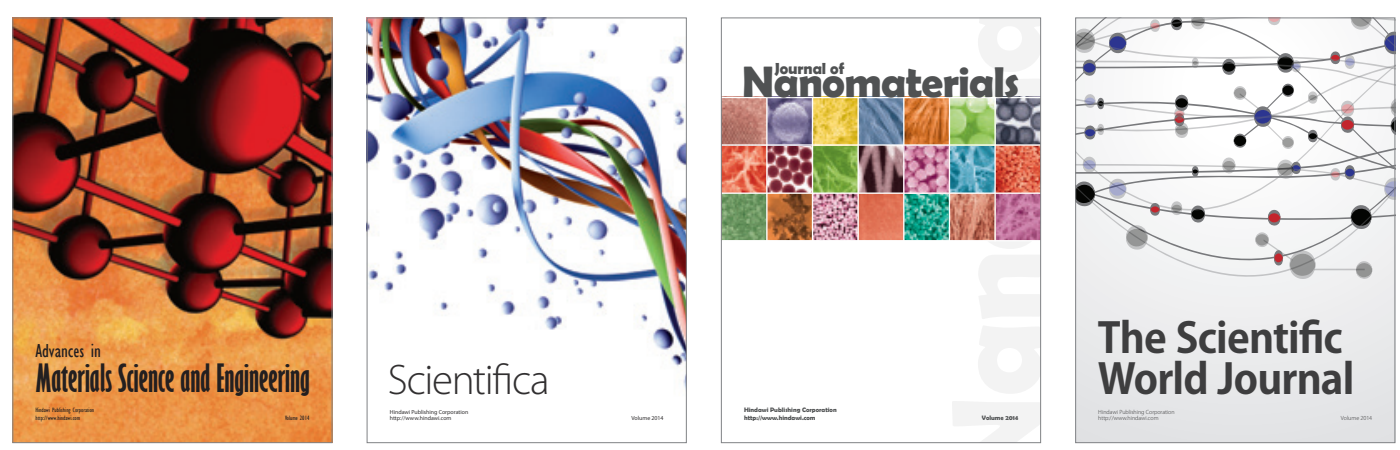

\section{The Scientific World Journal}
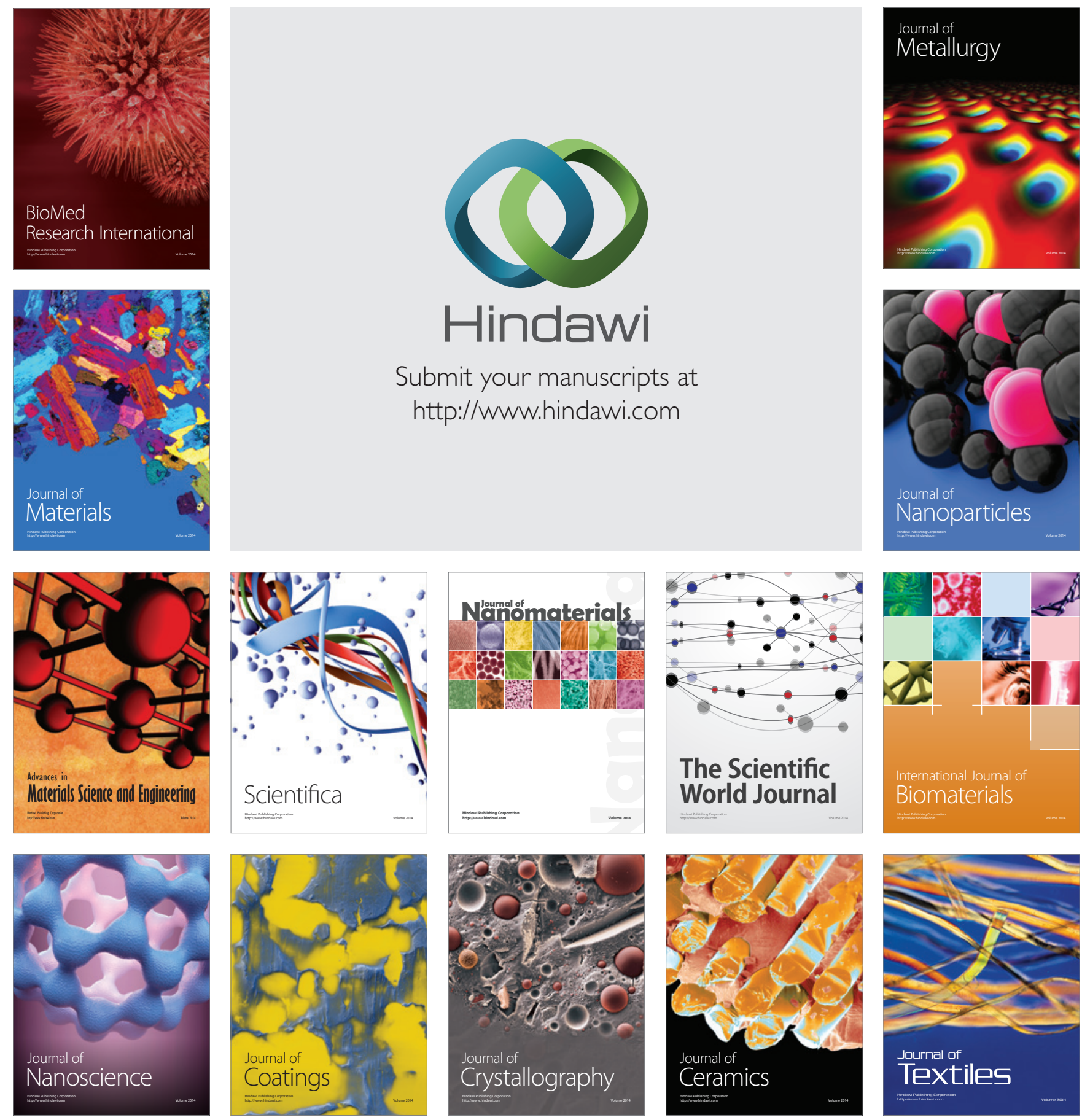